Produto \& Produção, vol. 12, n. 2, p. 87 - 107, jun. 2011

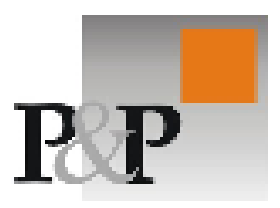

\title{
Priorização de requisitos para o desenvolvimento de software: uma abordagem multicritério utilizando o método AHP
}

Recebido em 08/02/2010. Aceito em 26/05/2011.

\section{André Luís Policani Freitas}

Professor Universidade Estadual do Norte Fluminense - UENF

Coordenador Laboratório de Engenharia de Produção - LEPROD

andrepolicani@yahoo.com

\section{Aline Gomes Cordeiro \\ Instituto Federal de Educação, Ciência e Tecnologia Fluminense \\ alinegomesc@yahoo.com.br}

Tendo em vista que um dos maiores riscos enfrentados por organizações que desenvolvem software está associado ao não atendimento das necessidades e expectativas dos usuários, este artigo tem como objetivo propor uma abordagem multicritério fundamentada no método AHP para priorização de requisitos do desenvolvimento de software. Desta forma, acredita-se que os requisitos priorizados podem servir como referência para as etapas que constituem o processo de desenvolvimento de software. Por meio de um estudo de caso, a abordagem proposta foi aplicada a um projeto de desenvolvimento de um software para gestão de recursos humanos. A partir dessa aplicação foi possível captar os julgamentos, determinar as prioridades dos requisitos segundo a percepção dos usuários e também verificar a consistência dos julgamentos.

Palavras-chave: Priorização de requisitos de software, desenvolvimento de software, AHP

Since one of the most risks faced by software development companies concerns to the no adequacy of users needs and expectative, this article proposes a multicriteria approach supported on the AHP method for prioritizing requirements of software development. In this context, we believe the prioritized requirements could be a reference to the subsequent steps of software development process. By conducting a case study, the proposed approach was applied to a software development project for human resource management. By doing so, users' evaluations were collected, the software requirements were prioritized according to their perspectives and the consistency analysis was performed.

Key-words: Software Requirements Prioritization, Software Development, AHP 


\section{INTRODUÇÃO}

Atualmente, nota-se que soluções de software, de uma forma geral, têm sido utilizadas nas organizações com o objetivo de apoiar as diversas atividades e a tomada de decisões. É possível observar a presença de softwares como ferramentas em diferentes ramos de negócios como saúde, educação e indústria. As melhorias obtidas a partir de sua utilização podem ser notadas e geralmente justificam os investimentos necessários.

Apesar disso, a atividade de desenvolvimento de software tem enfrentado algumas dificuldades. Para Karlsson e Ryan (1996) um dos maiores riscos enfrentados por organizações que desenvolvem software comercial está associado ao não atendimento das necessidades e expectativas dos usuários. Para esses autores, esse risco pode ocasionar danos na reputação, perda de pedidos e redução dos lucros da empresa.

Pesquisas que visam à identificação das causas para o problema acima citado apontam a fase de elicitação de requisitos, também conhecida como levantamento de requisitos, como preponderante para a melhoria do processo de desenvolvimento de software. A elicitação de requisitos é uma das atividades que ocorre no início do desenvolvimento de software. Erros gerados nesta atividade, se não são corrigidos, se estendem até o final do desenvolvimento e após a verificação de cada erro, todas as fases anteriores precisam ser refeitas.

Para Duan et al. (2009), alguns requisitos possuem maior impacto na satisfação dos usuários em relação ao software. Neste sentido, a entrega dos requisitos de acordo com a percepção dos usuários, além de contribuir para a satisfação desses usuários, pode ser essencial para a continuidade dos projetos de desenvolvimento.

Um dos métodos tradicionalmente utilizados para a priorização dos requisitos é o Método AHP (Analytic Hierarchy Process). Entretanto a literatura científica descreve que o emprego deste método para essa finalidade pode apresentar resultados satisfatórios (KARLSSON et al, 1998, KARATZAS et al., 2003, KARLSSON et al.,1996, LEE et al., 2008) ou insatisfatórios (LEHTOLA; KAUPPINEN, 2004).

Neste contexto, este artigo apresenta uma abordagem multicritério fundamentada no método AHP para priorização de requisitos do desenvolvimento de software. Por meio da aplicação desta abordagem realizada junto à equipe de desenvolvimento de um software para gestão de Recursos Humanos foi possível captar os julgamentos, determinar as prioridades dos requisitos segundo a percepção dos usuários e também verificar a consistência dos julgamentos.

De maneira sucinta este artigo está estruturado da seguinte forma: a seção 2 destaca a importância da elicitação e priorização de requisitos para o desenvolvimento de software, a seção 3 apresenta os principais aspectos relativos 
ao método AHP, destacando o emprego deste método em problemas decisórios com múltiplos avaliadores e também em problemas de priorização de requisitos, a seção 4 descreve a abordagem metodológica proposta para priorização, a seção 5 apresenta um estudo real no qual a abordagem proposta foi utilizada para priorização dos requisitos de um software de gestão de Recursos Humanos - os resultados obtidos são apresentados e analisados, e, finalmente, a seção 6 apresenta as considerações finais.

\section{PRIORIZAÇÃO DE REQUISITOS PARA AO DESENVOLVIMENTO DE SOFTWARE}

Um software deve possuir características que contribuam para a solução de problemas e melhoria da qualidade de trabalho dos usuários, tendo como conseqüência a melhoria da qualidade do serviço ou produto da empresa à qual o software se destina. Portanto, é preciso utilizar uma forma adequada de identificar e priorizar tais características, que constituem os requisitos.

A elicitação de requisitos tem sido reconhecida como uma das etapas determinantes para a qualidade de software. Para Larman (2004), requisitos são capacidades e condições às quais o sistema - e de forma mais ampla, o projeto deve atender.

Young (2004) afirma que requisitos são atributos necessários em um sistema para que ele tenha valor e utilidade para os clientes e usuários. Para o autor, os requisitos do sistema são importantes porque fornecem a base para todo o trabalho de desenvolvimento de software subseqüente.

Considerando que são as necessidades dos usuários que justificam o investimento em um projeto de software, não faz sentido que o foco principal do projeto seja outro senão a solução para essas necessidades. Embora essa seja uma afirmativa lógica, a realidade de muitos projetos de software mostra que é comum os objetivos do projeto se distanciarem das necessidades e desejos de seus usuários. Por isso, a fase de elicitação de requisitos pode ser compreendida como base para todas as outras atividades relativas ao desenvolvimento de software.

Os requisitos são itens que os usuários demandam porque possuem valor para eles. As prioridades são como eles esperam obtê-las considerando os diversos níveis de requisitos diante dos recursos limitados. Se os recursos fossem ilimitados, não haveria a necessidade de priorização (GILB; MAIER, 2005).

No entanto, os recursos necessários ao desenvolvimento de um software são limitados. Devido a restrições de tempo e orçamento, pode ser difícil implementar todos os requisitos identificados para um sistema. Os requisitos geralmente são implementados em etapas e a priorização ajuda a definir quais devem ser implementados prioritariamente (ALLEN et al., 2008).

Karlsson e Ryan (1996) e Berander (2004) apresentam visões semelhantes ao afirmarem que seleção do conjunto de requisitos é determinante para a satisfação dos usuários. Para Berander (2004) a seleção de forma "correta" dos requisitos que farão parte de cada versão é a etapa principal em direção ao sucesso de um projeto ou produto.

Por isso, de acordo com Duan et al. (2009), os desenvolvedores precisam ser cuidadosos na seleção do subconjunto dos requisitos que serão desenvolvidos. 
É necessário distinguir os requisitos que terão maior impacto para a satisfação dos usuários.

\section{AMD E O MÉTODO AHP}

O Auxílio Multicritério à Decisão (AMD) é um ramo da Pesquisa Operacional que objetiva fornecer ao decisor algumas ferramentas que permitam auxiliá-lo no tratamento de um problema decisório em que vários - e freqüentemente contraditórios - critérios e pontos de vista devem ser considerados (FREITAS, 2007). Nesse contexto, a abordagem AMD reconhece que, em geral, não existe uma alternativa que seja a melhor em todos os critérios. Conforme reportado por Vincke (1992), o termo "otimização" não deve ser mais utilizado pois, contrariando as técnicas clássicas da Pesquisa Operacional, os métodos de AMD não fornecem objetivamente as soluções ótimas. A solução encontrada será satisfatória, submetida a uma lógica matemática, com qualidade e custo aceitável. Por isso o uso do termo "auxílio" se faz conveniente.

Segundo Roy (1985) e corroborado por Vincke (1992), as abordagens de AMD podem ser classificadas como: abordagens de subordinação; abordagens do critério único de síntese; e abordagens interativas.

No âmbito dos Métodos de Subordinação, um conjunto finito de alternativas/ações $(A)$ são valoradas sobre uma família/vetor de critérios $(F)$, construindo-se relações de subordinação não compensatórias entre as alternativas. Os métodos da família ELECTRE e da família PROMETHEE são os métodos de subordinação mais difundidos cientificamente.

Segundo Antunes et al. (1989), as abordagens interativas alternam fases de cálculo com fases de decisão, nas quais o analista de decisão interage com o modelo, construindo a decisão mais adequada. Este tipo de abordagem é especialmente importante em situações em que se esteja buscando uma única solução que seja ótima ou que esteja próxima do ponto ótimo.

As abordagens do critério único de síntese caracterizam-se por buscar uma função que agregue diferentes funções de utilidade em uma função única. Os métodos que se baseiam nesta abordagem têm sido classificados como pertencentes à Escola Americana de métodos multicritério. Dentre os métodos e teorias que se baseiam nesta abordagem, são aqui destacados: a Teoria da Escolha Social (ARROW, 1963) e a Teoria da Utilidade Multiatributo (Multi-Attribute Utility Theory, MAUT), apresentada em Fishburn (1970) e consolidada em Keeney e Raiffa (1976); e o método AHP (SAATY, 1980).

Dentre a grande variedade de métodos de AMD atualmente existentes, o método AHP destaca-se por possibilitar a obtenção da importância relativa dos critérios considerados em problemas decisórios e a ordenação de tais critérios segundo essa importância. Esse raciocínio constitui a essência do problema de elicitação de requisitos no desenvolvimento de um software, fato que tem despertado o interesse de diversos pesquisadores atuantes nesta área do conhecimento, como no presente estudo. 


\subsection{Breve Descrição do Método AHP}

Proposto por Saaty no início dos anos 70, o método AHP objetiva a seleção/escolha de alternativas em um processo decisório que considere múltiplos critérios. Segundo Saaty (2000), o método AHP está estruturado segundo três princípios:

a) construção de hierarquias: sistemas complexos podem ser melhor compreendidos através do particionamento deste em elementos constituintes, estruturando tais elementos hierarquicamente e então sintetizando os julgamentos da importância relativa destes elementos em cada nível da hierarquia em um conjunto de prioridades (SAATY, 2000). Segundo este princípio, é preciso definir (figura 1): o foco principal (o objetivo do problema), os critérios/subcritérios (em tantos níveis quanto necessário), e as alternativas;

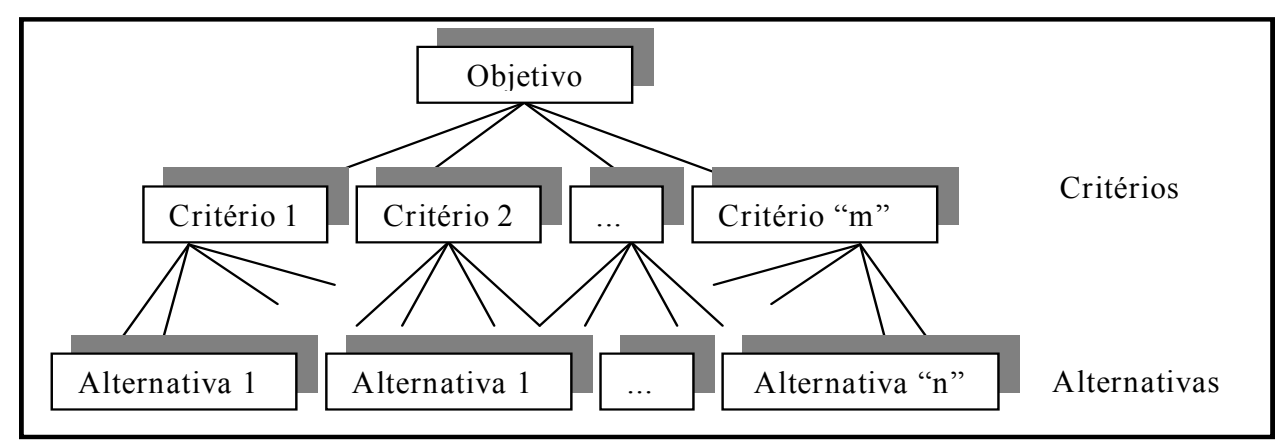

Figura 1 - Estrutura Hierárquica Básica

b) definição de prioridades: Segundo Saaty (2000), "o ser humano tem a habilidade de perceber as relações entre as coisas que observa, comparar pares de objetos similares à luz de certos critérios, e discriminar entre os membros de um par através do julgamento da intensidade de sua preferência de um elemento sobre o outro". Segundo Costa (2002), de forma sucinta, neste princípio é necessário cumprir as seguintes etapas:

- julgamentos paritários: julgar par a par os elementos de um nível da hierarquia à luz de cada elemento em conexão em um nível superior, compondo as matrizes de julgamento $A$ (através do uso das escalas apresentadas na tabela 1);

\begin{tabular}{lc}
\hline \multicolumn{1}{c}{ Escala Verbal } & Escala Numérica \\
\hline Igual preferência (importância) & 1 \\
Preferência (importância) fraca & 3 \\
Preferência (importância) moderada & 5 \\
Preferência (importância) forte & 7 \\
Preferência (importância) absoluta & 9 \\
$2,4,6,8$ são associadas a julgamentos intermediários & \\
\hline
\end{tabular}

Tabela 1 - Escalas de valor para julgamentos paritários

Fonte: Adaptado de Saaty (2000) 
A quantidade de julgamentos necessários para a construção de uma matriz de julgamentos genérica $A$ é $n(n-1) / 2$, onde $n$ é o número de elementos pertencentes a esta matriz. Os elementos de $A$ são definidos pelas condições:

$$
A=\left[\begin{array}{cccc}
1 & a_{12} & \cdots & a_{1 n} \\
1 / a_{21} & 1 & \cdots & a_{2 n} \\
\vdots & \vdots & \cdots & \vdots \\
1 / a_{n 1} & 1 / a_{n 2} & \cdots & 1
\end{array}\right], \text { onde: } \begin{aligned}
& a_{i j}>0 \Rightarrow \text { positiva } \\
& a_{i j}=1 \therefore a_{j i}=1 \\
& a_{i j}=1 / a_{j i} \Rightarrow \text { reciproca } \\
& a_{i k}=a_{i j} \cdot a_{j k} \Rightarrow \text { consistência }
\end{aligned}
$$

- Normalização das matrizes de julgamento: obtenção de quadros normalizados através da soma dos elementos de cada coluna das matrizes de julgamento e posterior divisão de cada elemento destas matrizes pelo somatório dos valores da respectiva coluna;

- Cálculo das prioridades médias locais (PML's): as PML's são as médias das linhas dos quadros normalizados;

- Cálculo das prioridades globais: nesta etapa deseja-se identificar um vetor de prioridades global (PG), que armazene a prioridade associada a cada alternativa em relação ao foco principal.

c) consistência lógica: o ser humano tem a habilidade de estabelecer relações entre objetos ou idéias de forma que elas sejam coerentes, tal que estas se relacionem bem entre si e suas relações apresentem consistência (SAATY, 2000). Assim o método AHP se propõe a calcular a Razão de Consistência dos julgamentos, denotada por $R C=I C / I R$, onde $I R$ é o Índice de Consistência Randômico obtido para uma matriz recíproca de ordem $n$, com elementos não-negativos e gerada randomicamente. O Índice de Consistência $(I C)$ é dado por $I C=\left(\lambda_{\text {máx }}-n\right) /(n-1)$, onde $\lambda_{\text {máx }}$ é o maior autovalor da matriz de julgamentos. Segundo Saaty (2000) a condição de consistência dos julgamentos é $R C \leq 0,10$.

\subsection{Inversão de Ordem no Emprego do Método AHP}

Uma das críticas mais freqüentes ao método AHP refere-se à ocorrência de um fenômeno denominado 'inversão de ordem' no emprego do método AHP tradicional. Este fenômeno é caracterizado pela possibilidade da alteração da ordem das prioridades globais das alternativas devido à remoção de uma alternativa ou introdução de uma nova alternativa ao problema.

Entretanto, segundo Schoner e Wedley (1989), a inversão de ordem não é simplesmente decorrente da introdução de uma nova alternativa, mas sim da introdução de uma nova alternativa sem a adequada reavaliação dos valores atribuídos aos elementos do nível hierárquico imediatamente superior. Além disso, Gomes, Araya e Carignano (2004) relatam que a ocorrência da inversão de ordem após a inclusão de uma nova alternativa pode ter sido ocasionada por falhas na etapa de modelagem.

Com o intuito de evitar a ocorrência deste problema, foram desenvolvidas três versões para o método AHP: o Método AHP Referenciado, proposto por Watson e Freeling (1982); o método AHP B-G, apresentado por Belton e Gear (1985); e, o método AHP Multiplicativo, proposto por Lootsma (1993). Apesar da relevância deste assunto, a investigação do emprego destes métodos quanto à eficácia no 
tratamento do fenômeno da inversão de ordem não está no contexto do presente trabalho.

\subsection{Uso do AHP em Problemas Decisórios com Múltiplos Avaliadores}

Nos últimos anos problemas decisórios complexos caracterizados pelo envolvimento de múltiplos avaliadores/decisores têm se tornado objeto de pesquisa no âmbito do Auxílio Multicritério à Decisão, uma das subáreas da Pesquisa Operacional. Segundo Van Den Honert (2001), frequentemente no grupo de decisores existe grande diferença em termos de formação profissional, competência e experiência no âmbito de um problema decisório. Além disso, nem todos os avaliadores/decisores têm o mesmo interesse na análise do problema, os critérios podem ser variados e essencialmente técnicos (avaliadores podem não estar habilitados a julgar à luz destes critérios), tornando evidente também que se os avaliadores/decisores não estiverem equitativamente qualificados para contribuir igualmente no processo decisório os resultados podem ser controversos.

Quando os avaliadores possuem os mesmos objetivos, Dyer e Forman (1999) relatam que o AHP pode ser utilizado em quatro contextos:

- Consenso: se os avaliadores têm (basicamente) os mesmos objetivos, é aconselhável que estes se reúnam e se esforcem para obter o consenso na estruturação do problema e nos julgamentos da importância relativa dos critérios e das alternativas;

- Votação: se o consenso não puder ser obtido em determinada situação, então o grupo de avaliadores pode realizar uma votação para escolher um julgamento intermediário;

- Uso de média geométrica: se o consenso não puder ser obtido e os membros não desejarem realizar uma votação, a média geométrica dos julgamentos dos membros pode ser calculada. Aczel e Saaty (1983) demonstraram que, quando a mesma importância é atribuída a todos avaliadores pertencentes a um grupo, o uso da média geométrica é a forma mais apropriada para sintetizar os julgamentos emitidos pelos diversos avaliadores.

- Modelos Distintos ou Avaliadores: se os avaliadores têm objetivos (ou pontos de vista) muito divergentes ou não podem se encontrar para discutir a decisão, cada avaliador pode emitir seus julgamentos separadamente. Os julgamentos de cada avaliador podem ser obtidos por:

- Modelos distintos: neste processo, cada avaliador atribui seus julgamentos em um modelo distinto (as prioridades resultantes podem ser obtidas pelo cálculo da média); e

- Avaliadores: na estrutura hierárquica é construído um "nível de avaliadores" abaixo do nível do objetivo principal. Os critérios e subcritérios são alocados em um nível abaixo do respectivo avaliador. Os critérios (e subcritérios) não necessariamente devem ser os mesmos para todos os avaliadores. Neste processo, atenção especial deve ser dada à atribuição de importância para cada avaliador - é usual atribuir a mesma importância a todos os avaliadores ou utilizar julgamentos paritários para obter a importância relativa entre os avaliadores.

Ramanathan e Ganesh (1994) propuseram a determinação da importância relativa dos avaliadores por meio de comparações paritárias interpessoais da 
importância ou influência entre os membros avaliadores (onde importância ou influência pode ser mensurada através de critérios como poder, experiência, habilidade de tumultuar, etc.) através da opinião de cada avaliador, utilizando o método AHP tradicional. Entretanto, segundo Van Den Honert (2001), este procedimento pode causar um desvio no processo decisório visto que há uma tendência de um indivíduo superestimar sua própria importância, especialmente se existe algo a ser ganho por ele.

Recentemente, Freitas et al. (2008) propuseram uma abordagem multicritério para auxílio à tomada de decisão fundamentada no algoritmo do método AHP para ser utilizada simultaneamente por vários grupos de avaliadores/decisores. Segundo os autores, apesar da ferramenta computacional desenvolvida para implementar a referida abordagem ter apresentado resultados satisfatórios no estudo de caso realizado, esta ainda encontra-se em fase de testes.

\subsection{Emprego do Método AHP para Priorização de Requisitos}

Na literatura científica é possível encontrar trabalhos favoráveis e trabalhos desfavoráveis ao emprego do método AHP para a priorização de requisitos para o desenvolvimento de software. Alguns desses trabalhos são apresentados a seguir.

Karlsson et al. (1998) destacam algumas características do AHP. Para estes autores, uma vez que a comparação é feita par a par, o esforço necessário pode ser muito grande. Em projetos de pequeno porte esse esforço pode ser aceitável, mas em projetos de desenvolvimento de grande porte a quantidade de comparações necessárias pode inviabilizar o uso do AHP.

Allen et al. (2008) citam o AHP ao listarem os métodos de priorização de requisitos. Segundo eles, o AHP é um método de apoio à decisão utilizado em situações nas quais múltiplos objetivos estão presentes. O método utiliza a comparação paritária para calcular o valor relativo de cada item (requisito). Os autores destacam a possibilidade de confirmação da consistência dos resultados como um aspecto positivo do método.

Segundo Karlsson et al. (1998), o AHP é bastante confiável visto que a redundância que existe nas comparações par a par reduz a ocorrência de erros de julgamento. Além disso, a comparação par a par força os usuários a analisar os requisitos de uma perspectiva diferente, possibilitando até mesmo a descoberta de erros na especificação da lista de requisitos.

Karatzas et al. (2003) utilizam o AHP para priorização dos requisitos de um sistema de gerenciamento de qualidade de ar urbano. De acordo com esses autores, estudos anteriores indicaram que fazer julgamentos relativos tende a ser mais rápido e mais confiável do que fazer julgamentos absolutos. Para eles o AHP é ideal para resolver problemas de natureza qualitativa, como é o caso dos requisitos dos usuários.

Karlsson et al. (1996) realizaram um estudo de caso para priorização dos requisitos por meio do AHP 14 requisitos foram selecionados para o estudo e 5 pessoas participaram da etapa da avaliação correspondente ao AHP Para os autores, devido ao fato do AHP basear-se em uma escala de razão, a diferença de prioridade entre os requisitos torna-se mais clara. Considerando como exemplo dois requisitos: A que equivale a $30 \%$ da prioridade total e $B$ que equivale a $10 \%$ da prioridade total, é possível afirmar que A é 3 vezes mais importante que $B$. 
Lee et al. (2008) utilizam AHP para definir a prioridade relativa dos requisitos necessários em sistemas voltados para a web. Para os autores, embora o AHP possa ser utilizado em outros contextos, o método se mostra satisfatório para a obtenção das prioridades relativas de requisitos.

Por outro lado, Lehtola e Kauppinen (2004) fornecem uma visão contrária ao uso do AHP ao destacarem alguns desafios encontrados durante uma aplicação do método:

a) Embora a definição do requisito mais importante na comparação par a par seja relativamente simples, os usuários acharam difícil estimar quanto um requisito é mais importante em relação a outro, mesmo utilizando uma escala de 5 pontos;

b) Comparações par a par com mais de 20 requisitos tornaram-se difíceis na prática, os usuários tiveram dificuldades em se concentrar nas comparações;

c) Requisitos de diferentes níveis de abstração causaram problemas;

d) Alguns usuários não aceitaram a comparação par a par. Eles consideraram que seria mais fácil selecionar os requisitos mais importantes ou colocar os requisitos em ordem descendente.

A partir dos autores citados nessa seção é possível afirmar que o AHP tem sido utilizado para priorização de requisitos apresentando resultados favoráveis e desfavoráveis ao seu emprego. No entanto, uma característica que deve ser observada é a dimensão do projeto de desenvolvimento do software.

\section{ABORDAGEM METODOLÓGICA PROPOSTA}

A abordagem metodológica proposta visando a utilização do método AHP para a priorização de requisitos em processos de desenvolvimento de software se constitui pelas seguintes etapas:

a) Identificar o conjunto $\boldsymbol{F}$ de critérios (requisitos) e agrupar esses critérios (atribuição de requisitos em grupos, por afinidade);

b) Utilizar o Método da Análise Hierárquica descrito na seção 3 para:

- Calcular a importância relativa dos grupos de requisitos $\left(\boldsymbol{W} \boldsymbol{G}_{\boldsymbol{i}}, \boldsymbol{i}=\mathbf{1}, \ldots, \boldsymbol{m}\right)$ : após a realização dos julgamentos paritários à luz do foco principal, calculamse as Prioridades Médias Locais, denotadas por $\boldsymbol{W G}_{i}$.

- Calcular a importância relativa dos requisitos $\left(W R_{i j}, i=1, \ldots, m\right.$ e $\left.j=1, \ldots, n\right)$ : após a realização dos julgamentos paritários dos critérios agrupados em cada grupo de requisitos, são calculadas as prioridades médias locais, denotadas por $\boldsymbol{W} \boldsymbol{R}_{i j}$.

- Calcular a importância real dos requisitos $\left(K \boldsymbol{R}_{j, j}, \boldsymbol{j}=\mathbf{1}, \ldots, n\right)$ : a partir dos valores calculados nos dois itens anteriores, calcula-se a importância real de cada requisito através da relação:

$$
K R_{j}=\sum_{u=1}^{t} W G_{i} \cdot W R_{i j} / t, i=1, \ldots, \text { me } j=1, \ldots, n
$$

- Calcular a Razão de Consistência para cada matriz de julgamentos.

É importante observar que esta abordagem não leva em consideração as alternativas a ordenar/priorizar, limitando-se à etapa de priorização dos critérios 
(requisitos) considerados no problema decisório. Entretanto é preciso determinar especificamente a importância real de cada requisito no âmbito do problema decisório, e não somente a importância relativa de cada requisito em relação ao elemento do nível superior da hierarquia ao qual está relacionado. Especificamente, este aspecto diferencia a abordagem proposta do tradicional algoritmo do método AHP.

\section{APLICAÇÃO DA ABORDAGEM PROPOSTA}

A abordagem proposta foi aplicada em um projeto de desenvolvimento de software de uma Fundação situada no município de Campos dos Goytacazes, localizado no Estado do Rio de Janeiro. Esta Fundação é mantenedora de algumas unidades de saúde vinculadas ao Sistema Único de Saúde (SUS). O objetivo do referido projeto é desenvolver um software que apóie as atividades relativas à gestão de Recursos Humanos (RH) da referida Fundação.

Os setores envolvidos no projeto de desenvolvimento possuem necessidades específicas em relação ao software e após algumas tentativas de aquisição de pacotes fechados de software, chegou-se à conclusão que essas necessidades específicas justificam o investimento em desenvolvimento. Entretanto, o software completo a ser desenvolvido deverá ser composto de vários Módulos integrados. Neste contexto, empregou-se a abordagem proposta para a priorização de requisitos que compõem o primeiro módulo, denominado Módulo $A$.

Visando obter informações e dados confiáveis, foi preciso buscar a participação de uma quantidade significativa de usuários que atuavam em funções e atividades relacionadas ao desenvolvimento do Módulo A. Participaram efetivamente deste estudo nove usuários - número considerado razoável, visto que no grupo de usuários havia pessoas-chave no processo e também porque as atividades de gestão hospitalar não poderiam ser totalmente interrompidas para 0 desenvolvimento do módulo em questão. Para tanto, foram implementadas as etapas descritas na seção anterior:

a) Identificação do conjunto $F$ de critérios (requisitos)

Os critérios da avaliação considerados neste estudo foram os requisitos para o desenvolvimento do software. Sendo assim, a primeira etapa consistiu na identificação, ou seja, na elicitação dos requisitos que fazem parte do escopo do projeto e devem estar presentes no software (Módulo A). A equipe de desenvolvedores utilizou quatro técnicas:

- Entrevista não estruturada: consistiu em perguntar aos usuários a respeito do assunto sem haver um preparo prévio de uma lista de perguntas;

- Entrevista estruturada: preparou-se uma lista de perguntas para fazer aos usuários;

- Prototipagem rápida: os usuários comentaram sobre um modelo em protótipo do sistema desejado;

- Análise de cenário: foi estabelecido um cenário, ou seja, uma descrição de uma seqüência de ações e eventos para um caso específico de alguma tarefa genérica que o sistema deve realizar. $O$ cenário incluiu vários casos de uso. 
As quatro técnicas listadas acima foram consideradas adequadas pela equipe de desenvolvimento, pois permitiram o entendimento do domínio onde o software se insere.

Por meio das entrevistas não estruturadas, os analistas puderam identificar algumas das funções básicas que o módulo A deveria possuir. Em seguida, entrevistas estruturadas foram realizadas com o objetivo de refinar as análises anteriores e resolver pequenas inconsistências.

A técnica de prototipagem permitiu que os clientes tivessem uma percepção mais aproximada de como o módulo A estava sendo desenvolvido e este protótipo possibilitou a identificação da necessidade de alguns ajustes. A análise de cenário foi considerada interessante para facilitar a compreensão das necessidades por parte de analistas e programadores do software em casos específicos.

A partir da aplicação das técnicas de elicitação utilizadas, a lista inicial de requisitos foi identificada. Para facilitar o entendimento da aplicação para os usuários, inicialmente os requisitos foram agrupados em relação ao contexto de aplicação. Como exemplo, requisitos relativos ao controle de freqüência dos funcionários foram inseridos em um mesmo grupo, o grupo 1. A tabela 2 apresenta a lista de requisitos agrupada.

\begin{tabular}{|c|c|}
\hline Grupo 1 & Controle de Freqüência dos Funcionários \\
\hline Requisito 1.1 & Registro de freqüência \\
\hline Requisito 1.2 & $\begin{array}{l}\text { Registro de informações de escalas e horários de } \\
\text { trabalho }\end{array}$ \\
\hline Requisito 1.3 & Emissão de relatório de freqüência \\
\hline Grupo 2 & Manutenção dos dados pessoais \\
\hline Requisito 2.1 & $\begin{array}{l}\text { Controle das informações pessoais dos } \\
\text { funcionários. }\end{array}$ \\
\hline Requisito 2.2 & $\begin{array}{l}\text { Registro de deficiências apresentadas pelos } \\
\text { funcionários }\end{array}$ \\
\hline Grupo 3 & Manutenção dos dados funcionais \\
\hline Requisito 3.1 & Emissão de crachás \\
\hline Requisito 3.2 & Manutenção de informações relativas à convocação \\
\hline Requisito 3.3 & $\begin{array}{l}\text { Controle de Alocação de funcionários em unidade e } \\
\text { setores }\end{array}$ \\
\hline Grupo 4 & Manutenção dos Afastamentos \\
\hline Requisito 4.1 & Controle de Férias \\
\hline Requisito 4.2 & $\begin{array}{l}\text { Controle de Afastamentos como Licenças Médicas } \\
\text { e INSS }\end{array}$ \\
\hline Grupo 5 & Movimentação de Documentos \\
\hline Requisito 5.1 & $\begin{array}{l}\text { Entrada e saída de carteiras de trabalho e } \\
\text { previdência social }\end{array}$ \\
\hline Requisito 5.2 & Solicitação e entrega de declarações \\
\hline
\end{tabular}


A partir da lista de requisitos agrupados a estrutura hierárquica para o problema em questão foi definida. Esta estrutura está representada pela figura 2.

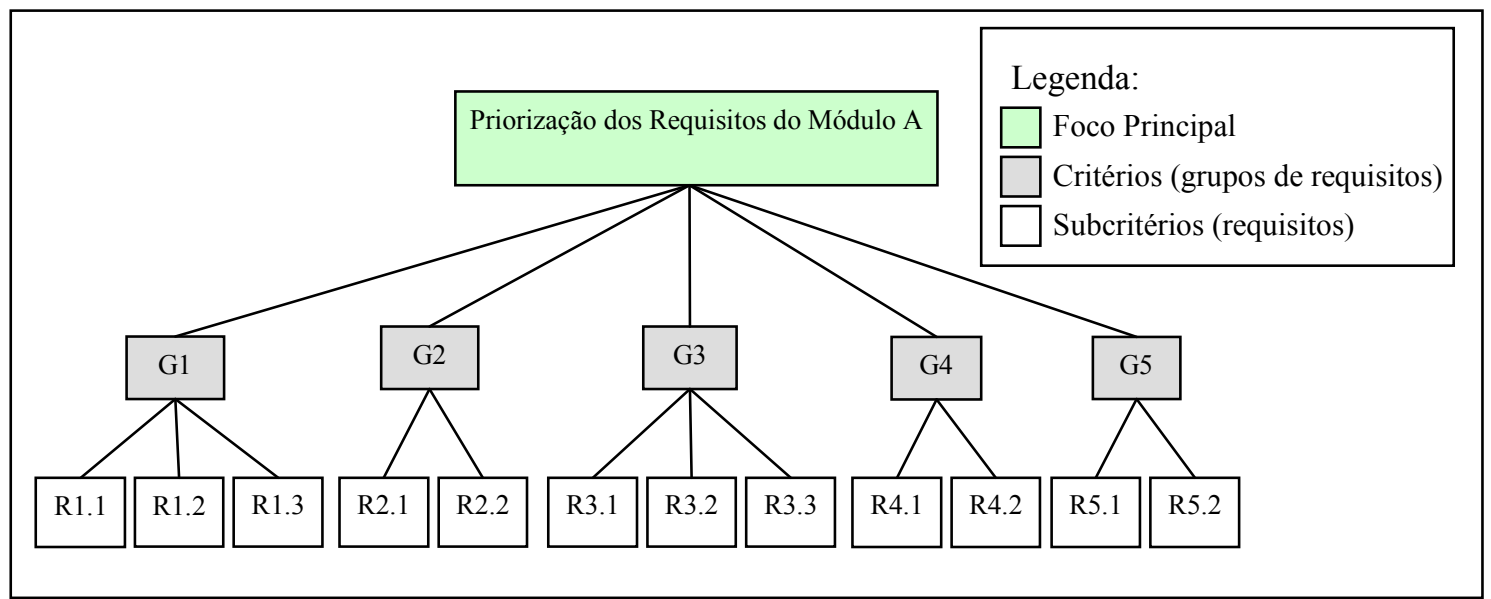

Figura 2 - Estrutura hierárquica para priorização dos requisitos

Ao observar a figura 2, nota-se que a estrutura hierárquica do problema tem início com a identificação do foco principal, que neste caso é a priorização dos requisitos do módulo $A$. Em seguida existe um segundo nível correspondente aos critérios definidos, composto por cinco grupos (G1, G2, G3, G4 e G5) de requisitos. O último nível da estrutura hierárquica refere-se aos subcritérios, ou seja, aos requisitos que compõem cada grupo.

Como o objetivo deste artigo é priorizar os requisitos, neste caso não existem alternativas a serem consideradas. A abordagem limita-se à priorização dos critérios e subcritérios.

c) Utilização do método AHP

Nesta etapa a equipe de desenvolvimento realizou a priorização da lista de requisitos obtida. Para que isso fosse possível, foi necessário desenvolver e aplicar o formulário de priorização. Os atributos que fizeram parte do formulário foram identificados na etapa de elicitação.

O formulário foi elaborado de forma que avaliação pudesse ser feita por cada usuário tendo como referência a escala de julgamento de valores proposta por Saaty (2000). Após a preparação, o formulário de priorização foi utilizado para captar os julgamentos dos nove usuários que atuavam no domínio onde o software (módulo A) seria implantado. Neste caso, os formulários foram disponibilizados para que fossem preenchidos individualmente, evitando que a opinião de um usuário interferisse nos julgamentos dos demais. O formulário aplicado encontra-se no Anexo deste artigo.

Para implementação do algoritmo do método AHP, utilizou-se o software IPE (COSTA, 2010). Os resultados obtidos em cada etapa definida na abordagem proposta são apresentados a seguir.

- Cálculo da importância relativa dos grupos de requisitos $\left(W G_{i}\right)$ : A tabela 3 apresenta a importância relativa dos grupos de requisitos e a ordenação correspondente, segundo a percepção de cada usuário. É importante observar que o grupo G5 foi considerado mais importante por cinco dos nove usuários. Mas, por 
outro lado, o grupo G5 também foi considerado como o menos importante por três usuários. À primeira vista, tal resultado indica que os usuários apresentam percepções muito diferenciadas a respeito da importância dos grupos de requisitos para o desenvolvimento do módulo $A$.

Entretanto, considerando a média geométrica dos julgamentos - procedimento recomendado por Aczel e Saaty (1983) para obtenção de um vetor de prioridades que represente os julgamentos de todos os usuários pertencentes ao grupo de avaliadores - notou-se que o grupo G3 (Manutenção dos dados funcionais) apresenta a maior importância relativa média $(\boldsymbol{M G}=0,299)$. Os grupos $\mathbf{G 5}$ (Movimentação de Documentos) e G1 (Controle de freqüência dos funcionários) obtiveram importância relativa muito próximas, tecnicamente de igual prioridade.

\begin{tabular}{|c|c|c|c|c|c|c|}
\hline & & G1 & $\mathbf{G 2}$ & G3 & G4 & G5 \\
\hline \multirow{2}{*}{ Usuário 1} & $\overline{W G_{i}}$ & 0,186 & 0,172 & 0,096 & 0,039 & 0,507 \\
\hline & Ordem & $2 .^{\circ}$ & $3 .^{\circ}$ & $4 .^{\circ}$ & $5 .^{\circ}$ & $1 .^{\circ}$ \\
\hline \multirow[b]{2}{*}{ Usuário 2} & $W G_{i}$ & 0,118 & 0,164 & 0,472 & 0,157 & 0,088 \\
\hline & Ordem & $4 .^{\circ}$ & $2 .^{\circ}$ & $1 .^{\circ}$ & $3 .^{\circ}$ & $5 .^{\circ}$ \\
\hline \multirow[b]{2}{*}{ Usu } & $W G_{i}$ & 0,511 & 0,079 & 0,232 & 0,149 & 0,030 \\
\hline & Ordem & $1 .^{\circ}$ & $4 .^{\circ}$ & $2 .^{\circ}$ & $3 .^{\circ}$ & $5 .^{\circ}$ \\
\hline \multirow{2}{*}{ Usuá } & $W G_{i}$ &, 093 &, 033 & 0,14 & 0,365 & 0,365 \\
\hline & Ordem & $3 .^{\circ}$ & $4 .^{\circ}$ & $2 .^{\circ}$ & $1 .^{\circ}$ & $1 .^{\circ}$ \\
\hline \multirow{2}{*}{ Usuário 5} & $W G_{i}$ & 0,126 & 0,123 & 0,232 & 0,250 & 0,268 \\
\hline & Ordem & $4 .^{\circ}$ & $5 .^{\circ}$ & $3 .^{\circ}$ & $2 .^{\circ}$ & $1 .^{\circ}$ \\
\hline \multirow{2}{*}{ Us } & $W G_{i}$ & 0,200 & 0,200 & 0,20 & 0,200 & 0,200 \\
\hline & Ordem & $1 .^{\circ}$ & $1 .^{\circ}$ & $1 .^{\circ}$ & $1 .^{\circ}$ & $1 .^{\circ}$ \\
\hline \multirow{2}{*}{ Usuário 7} & $W G_{i}$ & 0,028 & 0,112 & 0,242 & 0,062 & 0,556 \\
\hline & Orde & $5 .^{\circ}$ & $3 .^{\circ}$ & $2 .^{\circ}$ & $4 .^{\circ}$ & $1 .^{\circ}$ \\
\hline \multirow{2}{*}{ Us } & $W G_{i}$ & 0,296 & 0,175 & 0,445 & 0,051 & 0,034 \\
\hline & Ordem & $2 .^{\circ}$ & $3 .^{\circ}$ & $1 .^{\circ}$ & $4 .^{\circ}$ & $5 .^{\circ}$ \\
\hline \multirow{4}{*}{ Usuá } & $W G_{i}$ & 0,294 & 0,315 & 0,294 & 0,045 & 0,052 \\
\hline & Orde & 2. & $1 .^{\circ}$ & 2. & $4 .^{\circ}$ & $3 .^{\circ}$ \\
\hline & $M G_{i}$ &, 197 & 0,166 & 0,299 & 0,140 & 0,198 \\
\hline & Ordem & $3 .^{\circ}$ & $4 .^{\circ}$ & $1 .^{\circ}$ & $5 .^{\circ}$ & $2 .^{\circ}$ \\
\hline
\end{tabular}

Tabela 3 - Importância relativa dos grupos de requisitos Fonte: Resultados da pesquisa.

- Cálculo da importância relativa dos requisitos $\left(W R_{i j}\right)$ : A tabela 4 apresenta a importância relativa de cada requisito dentro do grupo ao qual pertence, segundo cada usuário. Em termos do grupo G1, a média geométrica dos julgamentos indicou que o requisito R1.3 foi o de maior prioridade. Para o grupo $\mathrm{G} 2$ houve uma quase unanimidade da maior importância para o requisito R2.1 (apenas um usuário considerou os requisitos deste grupo igualmente importantes). Em relação ao grupo G3, a média geométrica dos julgamentos revelou que o requisito R.3.3 foi considerado o mais importante (embora alguns usuários não tenham considerado este requisito como o mais importante). A análise dos resultados dos grupos G4 e G5 revelam uma tendência dos usuários considerarem os respectivos requisitos igualmente importantes (os valores da média geométrica dos julgamentos confirmam 
este fato), embora alguns usuários tenham indicado a maior importância relativa para um dos requisitos.

\begin{tabular}{c|ccc|cc|ccc|cc|cc}
\hline & \multicolumn{3}{|c|}{ Grupo 1 } & \multicolumn{2}{|c|}{ Grupo 2 } & \multicolumn{3}{c|}{ Grupo 3 } & \multicolumn{2}{c|}{ Grupo 4 } & \multicolumn{2}{c}{ Grupo 5 } \\
\hline & $\mathbf{R 1 . 1}$ & $\mathbf{R 1 . 2}$ & $\mathbf{R 1 . 3}$ & $\mathbf{R 2 . 1}$ & $\mathbf{R 2 . 2}$ & $\mathbf{R 3 . 1}$ & $\mathbf{R 3 . 2}$ & $\mathbf{R 3 . 3}$ & $\mathbf{R 4 . 1}$ & $\mathbf{R 4 . 2}$ & $\mathbf{R 5 . 1}$ & $\mathbf{R 5 . 2}$ \\
\hline $\begin{array}{c}\text { Usuário } \\
\mathbf{1}\end{array}$ & 0,701 & 0,065 & 0,234 & 0,875 & 0,125 & 0,701 & 0,065 & 0,234 & 0,125 & 0,875 & 0,875 & 0,125 \\
\hline $\begin{array}{c}\text { Usuário } \\
\mathbf{2}\end{array}$ & 0,089 & 0,658 & 0,253 & 0,889 & 0,111 & 0,311 & 0,066 & 0,623 & 0,167 & 0,833 & 0,833 & 0,167 \\
\hline $\begin{array}{c}\text { Usuário } \\
\mathbf{3}\end{array}$ & 0,715 & 0,062 & 0,223 & 0,889 & 0,111 & 0,058 & 0,235 & 0,707 & 0,833 & 0,167 & 0,167 & 0,833 \\
\hline $\begin{array}{c}\text { Usuário } \\
\mathbf{4}\end{array}$ & 0,343 & 0,324 & 0,333 & 0,833 & 0,167 & 0,071 & 0,206 & 0,723 & 0,500 & 0,500 & 0,500 & 0,500 \\
\hline $\begin{array}{c}\text { Usuário } \\
\mathbf{5}\end{array}$ & 0,333 & 0,333 & 0,333 & 0,875 & 0,125 & 0,333 & 0,333 & 0,333 & 0,500 & 0,500 & 0,500 & 0,500 \\
\hline $\begin{array}{c}\text { Usuário } \\
\mathbf{6}\end{array}$ & 0,333 & 0,333 & 0,333 & 0,500 & 0,500 & 0,685 & 0,068 & 0,247 & 0,500 & 0,500 & 0,500 & 0,500 \\
\hline $\begin{array}{c}\text { Usuário } \\
\mathbf{7}\end{array}$ & 0,075 & 0,683 & 0,242 & 0,900 & 0,100 & 0,206 & 0,071 & 0,723 & 0,875 & 0,125 & 0,167 & 0,833 \\
\hline $\begin{array}{c}\text { Usuário } \\
\mathbf{8}\end{array}$ & 0,206 & 0,071 & 0,723 & 0,875 & 0,125 & 0,515 & 0,178 & 0,307 & 0,500 & 0,500 & 0,875 & 0,125 \\
\hline $\begin{array}{c}\text { Usuário } \\
\mathbf{9}\end{array}$ & 0,467 & 0,067 & 0,467 & 0,833 & 0,167 & 0,111 & 0,111 & 0,778 & 0,500 & 0,500 & 0,500 & 0,500 \\
\hline $\mathbf{M G}$ & 0,312 & 0,240 & 0,449 & 0,847 & 0,153 & 0,278 & 0,163 & 0,559 & 0,500 & 0,500 & 0,563 & 0,437 \\
\hline \multicolumn{1}{c}{ Tabela 4- Importância relativa dos requisitos em cada grupo } & & &
\end{tabular}

Entretanto, para determinar quais foram os requisitos mais importantes de toda a lista de requisitos, requisitos estes considerados de maior prioridade para 0 desenvolvimento do módulo $A$, foi necessário calcular a importância real dos requisitos. Estes resultados foram obtidos na etapa seguinte.

- Cálculo da importância real dos requisitos $\left(K R_{j}\right)$ : Uma vez obtida a importância relativa de cada critério (grupo de requisitos) à luz do Foco Principal (resultados apresentados na tabela 3) e importância relativa de cada subcritério (requisito) à luz de cada critério (resultados apresentados na tabela 4) para cada usuário, a importância real $\left(K R_{j}\right)$ de cada subcritério pode ser obtida, fazendo-se o somatório dos produtos da importância deste subcritério à luz de cada critério pela importância relativa do critério correspondente, à luz do Foco Principal e dividindo-se pelo total de usuários participantes da avaliação. Os resultados obtidos são apresentados na tabela 5. A importância real de cada requisito foi obtida da seguinte forma:

Onde:

$$
K R_{j}=\sum_{u=1}^{t} W G_{i} \cdot W R_{i j} / t
$$

$K R_{j}$ : prioridade real de cada requisito $j$;

$u$ : usuário $(u=1,2, \ldots, t)$; 
$W G_{i}$ : importância relativa de cada grupo $i$ de requisitos;

$W R_{i j}$ : importância relativa de cada requisito $j$ do grupo $i$;

$\begin{array}{llllllllllll}\text { R1.1 } & \text { R1.2 } & \text { R1.3 } & \text { R2.1 } & \text { R2.2 } & \text { R3.1 } & \text { R3.2 } & \text { R3.3 } & \text { R4.1 } & \text { R4.2 } & \text { R5.1 } & \text { R5.2 }\end{array}$

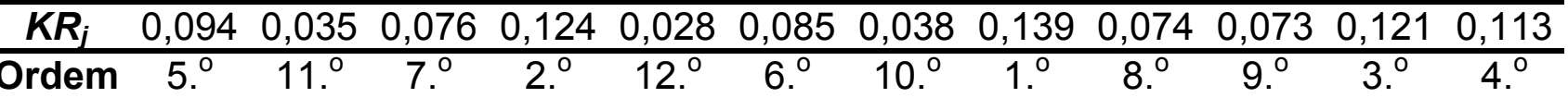

Tabela 5 - Prioridade real de cada requisito

Fonte: Resultados da pesquisa.

Considerando os resultados da tabela 5 , constatou-se que o requisito de maior prioridade segundo o julgamento dos usuários, foi o requisito R3.3 com prioridade real igual a 0,139 . O requisito $\mathrm{R} 2.2$ foi considerado o menos prioritário, com valor de prioridade real igual a 0,028 .

- Cálculo da Razão de Consistência para cada matriz de julgamentos: Segundo Saaty (2000), no método AHP é possível determinar o grau de inconsistência dos julgamentos paritários realizados avaliando a intensidade com que o maior autovalor da matriz de julgamentos se afasta de sua ordem. O software utilizado na análise dos resultados (IPÊ) forneceu os valores relativos à razão de consistência para as matrizes de julgamentos, esses valores são apresentados na tabela 6 .

\begin{tabular}{ccccccc}
\hline & Foco & G1 & G2 & G3 & G4 & G5 \\
\hline Usuário 1 & 0,379 & 0,406 & 0,000 & 0,406 & 0,000 & 0,000 \\
\hline Usuário 2 & 1,068 & 0,265 & 0,000 & 0,119 & 0,000 & 0,000 \\
\hline Usuário 3 & 0,433 & 0,233 & 0,000 & 0,406 & 0,000 & 0,000 \\
\hline Usuário 4 & 0,349 & 4,972 & 0,000 & 0,270 & 0,000 & 0,000 \\
\hline Usuário 5 & 0,549 & 0,000 & 0,000 & 0,000 & 0,000 & 0,000 \\
\hline Usuário 6 & 0,000 & 0,000 & 0,000 & 0,215 & 0,000 & 0,000 \\
\hline Usuário 7 & 0,315 & 0,336 & 0,000 & 0,270 & 0,000 & 0,000 \\
\hline Usuário 8 & 0,434 & 0,270 & 0,000 & 0,326 & 0,000 & 0,000 \\
\hline Usuário 9 & 0,004 & 0,000 & 0,000 & 0,000 & 0,000 & 0,000 \\
\hline
\end{tabular}

Tabela 6 - Razões de Consistência (RC) dos julgamentos segundo cada usuário Fonte: Resultados da pesquisa.

Segundo Saaty (2000) a condição de consistência dos julgamentos é $R C \leq$ 0,10 . Ao observar os dados apresentados na tabela 6 , notou-se que parte significativa dos julgamentos foi considerada inconsistente ( $R C>0,10)$. Com exceção do usuário 9 , todos os usuários apresentaram ao menos uma matriz de julgamentos considerada inconsistente. Ou seja, no presente estudo as prioridades obtidas para os requisitos por meio do emprego da abordagem proposta não podem ser consideradas satisfatórias para fins de auxílio à tomada de decisão.

Entretanto, considerando as etapas da abordagem proposta e os resultados apresentados neste estudo, buscou-se identificar possíveis causas que podem ter contribuído para a ocorrência de julgamentos inconsistentes, dentre as quais citamse: 
- Dificuldade em estabelecer a importância relativa dos requisitos: sob este aspecto, pode não ter havido um perfeito entendimento do processo de julgamento de valores de forma paritária, por meio do emprego da escala de Saaty (2000). Neste estudo, alguns usuários atribuíram o valor 1 na comparação entre os pares de requisitos, fato que pode representar uma dificuldade em estabelecer a comparação do par de requisitos em relação à importância.

- Nova etapa no processo de desenvolvimento de software (priorização de requisitos): O processo de priorização de requisitos após a elicitação geralmente não é realizado pela equipe de desenvolvimento do software em questão, ou caso isso seja realizado, o processo ocorre de forma intuitiva. Desta forma, a priorização de requisitos de forma estruturada é uma atividade nova para os usuários participantes deste processo;

- Quantidade excessiva de julgamentos: A quantidade de julgamentos necessários ao uso do método AHP é definida pela expressão $n(n-1) / 2$, onde $\mathrm{n}$ é o número de critérios (requisitos) utilizados. Apesar de ter sido realizado em um projeto de desenvolvimento de software de pequeno porte, no estudo foram utilizados cinco grupos de requisitos (resultando em 10 comparações paritárias) e 12 requisitos (considerando os cinco grupos de requisitos, nove julgamentos paritários foram gerados), totalizando 19 julgamentos paritários realizados por cada avaliador. Considerando as duas possíveis causas destacadas anteriormente, 19 julgamentos paritários podem representar um número significativamente grande para estes usuários. Um procedimento que pode contribuir para a atenuação deste problema seria definir o(s) grupo(s) de requisitos que cada usuário deve avaliar. Neste caso, a quantidade de comparações seria menor e talvez o processo possa exigir um esforço cognitivo menor dos usuários.

- Conhecimento ou expertise dos usuários: o emprego do método AHP como ferramenta de apoio à decisão pressupõe que os avaliadores detêm conhecimento significativo do processo decisório e das variáveis envolvidas. No presente estudo, não foi apurado precisamente o tempo de experiência de cada usuário participante nas atividades relacionadas ao módulo $A$.

Além dos aspectos supracitados, uma das premissas presentes no processo de desenvolvimento de software diz respeito à característica mutável dos requisitos. Por diversos motivos os requisitos definidos inicialmente para um determinado projeto de desenvolvimento podem ser alterados. O método AHP possibilita a priorização de forma relativa e, por isso, se um novo requisito for identificado, todo o processo de priorização precisa ser refeito. Conforme relatado por Lehtola e Kauppinen (2004), este aspecto particular pode causar insatisfação dos usuários participantes do processo de desenvolvimento de software. Em geral, métodos absolutos permitem a redefinição das prioridades de forma mais simples.

Diante do exposto, visando contribuir para a atividade de priorização de requisitos por meio da utilização da abordagem proposta, recomenda-se que antes de uma aplicação técnica e profissional da abordagem proposta seja realizado um exercício ilustrativo do emprego do método AHP, mesmo que este exercício aborde um problema decisório não diretamente relacionado à priorização dos requisitos. Neste exercício, os princípios do método AHP seriam apresentados de maneira lúdica, para facilitar a compreensão dos eventuais avaliadores. Maior ênfase deve ser dada ao entendimento da estrutura hierárquica $e$, principalmente à compreensão da escala de julgamento de valor e a sua utilização por meio do instrumento de coleta de dados (formulário para priorização de requisitos). A apresentação do 
conteúdo algébrico que envolve o algoritmo do método AHP pode ser uma etapa opcional, mas em geral não necessária.

\section{CONSIDERAÇÕES FINAIS}

Estudos recentes destacados neste artigo revelam que a elicitação e priorização de requisitos são etapas muito importantes na busca pelo sucesso de projetos de desenvolvimento de software, pois por meio destas atividades todas as demais atividades do desenvolvimento do projeto são direcionadas. Ressaltou-se também que essas atividades devem ser preferencialmente realizadas com a participação e contribuição de pessoas que atuam nos processos que são objeto do projeto de software a ser desenvolvido. Este fato revela que a elicitação e priorização de requisitos constituem um problema decisório com múltiplos critérios no qual múltiplos avaliadores estão envolvidos, sendo que tais avaliadores em geral apresentam percepções distintas sobre o problema.

Entretanto, em problemas desta natureza é necessário obter uma solução (no caso em questão, um vetor das prioridades dos requisitos de software) que melhor represente a percepção do grupo de avaliadores. Um dos métodos de auxílio à tomada de decisão mais utilizados para este fim denomina-se AHP (Analytic Hierarchy Process). Porém existem estudos científicos que relatam resultados satisfatórios e insatisfatórios do emprego deste método em problemas de priorização de requisitos.

Desejando contribuir para esta problemática, este artigo apresentou uma abordagem metodológica fundamentada no método AHP com a finalidade de priorizar requisitos a partir da percepção dos usuários participantes do processo de desenvolvimento de software. Por meio de um estudo real, a referida abordagem foi utilizada no desenvolvimento de um módulo (Módulo A) de um projeto software de apoio à gestão de Recursos Humanos $(\mathrm{RH})$. Após elicitação dos requisitos e posterior julgamento de valores e priorização dos requisitos, os resultados obtidos não foram considerados satisfatórios porque os valores referentes à Razão de Consistência para os diversos usuários indicaram que houve inconsistência nos julgamentos.

Entretanto, considera-se que os resultados insatisfatórios obtidos encontram-se no âmbito do estudo em questão, não sendo suficientes para concluir que a abordagem proposta e o método AHP não sejam adequados para priorizar requisitos de software. Neste contexto, alguns aspectos que poderiam ter contribuído para a inconsistência dos julgamentos foram relatados neste artigo e podem ser relevantes em outros processos de priorização de requisitos de software.

Adicionalmente, recomenda-se que os princípios do método AHP sejam apresentados e exercitados de maneira lúdica junto aos usuários, antes de utilizar a abordagem proposta para priorização de requisitos. Atenção especial deve ser destinada à compreensão da estrutura hierárquica e, principalmente ao entendimento e uso da escala de julgamento de valores por meio do instrumento (formulário) de coleta de dados.

\section{REFERÊNCIAS}


ACZEL, J.; SAATY, T. L. Procedures for synthesizing ratio judgments, Journal of Mathematical Psychology. v.27, p. 93-102, 1983.

ALLEN, J.H., et al. Software Security Engineering: A Guide for Project Managers. Addison Wesley Professional, 2008. 368p.

ANTUNES, H. A.; ALVES, M. J.; SILVA, A. L.; CLÍMACO, J. N. Algumas reflexões sobre uma base de métodos de programação linear multicritério. Investigação Operacional, v. 9, n. 2, p. 19-35. 1989.

ARROW, K. J. Social Choice and Individual Values. London: JohnWiley and Sons, 1963.

BELTON, V.; GEAR, A. E. The legitimacy of rank reversal - a comment. Omega, n.13, 3: p. 143-144, 1985.

BERANDER, P. Prioritization of Stakeholder Needs in Software Engineering Understanding and Evaluation. Thesis (degree of Licentiate of Technology in Software Engineering), Sweden, Department of Systems and Software Engineering Blekinge Institute of Technology, 172p, 2004.

COSTA, H. G. Introdução ao Método de Análise Hierárquica - Análise Multicritério no Auxílio à Decisão, Niterói, Biblioteca da Escola de Engenharia e Instituto de Computação da UFF, 2002.

COSTA, H. G. Software IPÊ - Página de download do sistema. Disponível em: http://www.professores.uff.br/helder/Arquivo\%20IPE1.htm. Acesso em 21 de junho de 2010.

DUAN, C. et al. Towards automated requirements prioritization and triage. Requirements Engineering, v. 14, p. 73-89, 2009.

DYER, R. F.; FORMAN, E. H. Group decision support with the Analytic Hierarchy Process, Decision Support Systems. v.8. p. 99-124. 1999.

FISHBURN, P. C. Utility theory for decision making. New York: Wiley, 1970.

FREITAS, A. L. P. Uma abordagem multicritério para a classificação de hotéis. RAUSP. Revista de Administração da USP, v. 42, n. ${ }^{\circ}$ 3, Jul.-Set., p. 338-348. 2007.

FREITAS, A. L. P.; TREVIZANO, W. A.; COSTA, H. G. Uma abordagem multicritério para problemas decisórios com múltiplos grupos de avaliadores. Investigação Operacional, v. 28, p. 133-149, 2008.

GILB, T.; MAIER, M. W. Managing Priorities: A Key to Systematic Decision-Making. In: INCOSE CONFERENCE, Rochester, 2005. Proceedings of Incose Conference, Rochester, NY USA, 2005. 
GOMES, L. F. A. M.; ARAYA, M. C. G; CARIGNANO, C. Tomada de decisões em cenários complexos: introdução aos métodos discretos do apoio multicritério à decisão. Thompson. 168 p. 2004.

KARATZAS, K.; DIOUDI, E.; MOUSSIOPOULOS, N. Identification of major components for integrated urban air quality management and information systems via user requirements prioritization. Environmental Modelling \& Software. v. 18, p. 173-178, 2003.

KARLSSON, J. Software Requirements Prioritizing. In: ICRE '96, 1996. Proceedings of International Conference on Requirements Engineering, 1996, p. 110-116.

KARLSSON, J.; RYAN, K. Supporting the selection of Software Requirements. In: 8th INTERNATIONAL WORKSHOP ON SOFTWARE SPECIFICATION AND DESIGN, 1996. Proceedings of the 8th International Workshop on Software Specification and Design (IWSSD '96), 1996, p. 146-149.

KARLSSON, J.; WOHLIN, C.; REGNELL, B. An evaluation of methods for prioritizing software requirements. Information and Software Technology, v. 39, p. 939-947, 1998.

KEENEY, R. L.; RAIFFA, H. Decisions with Multiple Objectives: preferences and value tradeoffs. New York: John Willey \& Sons, 569 p., 1976.

LARMAN, C. Utilizando UML e padrões: uma introdução à análise e ao projeto orientado a objetos e ao Processo Unificado. 2. ed. Porto Alegre: Bookman, 2004. $607 p$.

LEE, K.; JOSHI, K.; BAE, M. Using analytical hierarchy Process (AHP) to identify the relative importance of the features needed for web-based systems development. Information Resources Management Journal, v. 21, n. 3, p. 88-100, 2008.

LEHTOLA, L.; KAUPPINEN, M. Empirical Evaluation of Two Requirements Prioritization Methods in Product Development Projects. In: EUROPEAN SOFTWARE PROCESS IMPROVEMENT CONFERENCE, 2004. Proceedings of the European Software Process Improvement Conference (EuroSPI 2004), 2004, p. 161170.

LOOTSMA, F. A. Scale Sensitivity in the multiplicative AHP and SMART, Journal of Multicriteria Decision Analysis, n.2. p. 87-110. 1993.

RAMANATHAN, R.; GANESH, L. S. Group preference aggregation methods employed in AHP: An evaluation and an intrinsic process for deriving members' weightages, European Journal of Operational Research, n.79. p. 249-265. 1994.

ROY, B. Mèthodologie Multicritère d'Àide à la Dècision, Economica, Paris, 1985.

SAATY, T. L. The Analytic Hierarquic Process. Pittsburg: RWS Publications, 1980.

SAATY, T. L. Decision making for leaders. Pittsburg, USA: WS. Publications, 2000. 
SCHONER, B.; WEDLEY, W. C. Ambiguous Criteria Weights in AHP: Consequences and Solutions. Decision Sciences, n.20. p. 462-475. 1989.

VAN DEN HONERT, R. C. Decisional Power in Group Decision Making: A Note on the Allocation of Group Member's Weight in the Multiplicative AHP and SMART. Group Decision and Negociation, n.10. p. 275-286, 2001.

VINCKE, P. Multicriteria decision Aid. New York: John Wiley, 1992.

WATSON, S. R.; FREELING, A. N. S. Comment on: Assessing attribute weights by ratios. Omega, 11, 1982.

YOUNG, R. R. The Requirements Engineering Handbook. Boston: Artech House, 2004. 251p.

Agradecimentos

Os autores agradecem 0 apoio fornecido pelo Conselho Nacional de Desenvolvimento Científico e Tecnológico (CNPq) e aos funcionários da Fundação Dr. João Barcellos Martins (FJBM), pelos julgamentos e contribuições ao estudo descrito neste artigo. 


\section{Anexo: Formulário de Priorização dos Requisitos utilizando AHP}

\section{APA UENF Mestrado PPGEP-UENF}

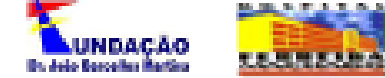

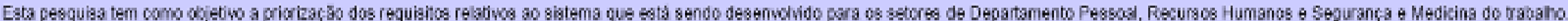

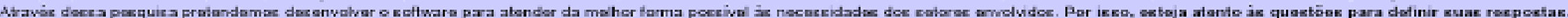
Funç̄o: Tempo de Experiéncia no setor:

1) Preencha os itens a seguir utilizando a coluna da esquerda para indicar qual requisito é mais importante de acordo com a sua opiniäo e a da direita para indicar a intensidade da importância do primeiro em relaçäo ao segundo requisito.

\begin{tabular}{|c|c|c|c|c|c|c|c|c|c|c|}
\hline \multirow{2}{*}{\multicolumn{2}{|c|}{ IMPORT TANCIA (marque em cada timha qual dos dois vecé considera mass importante) }} & \multicolumn{9}{|c|}{ JULGAMENTO (ondique a hrensitade de mpartincig) } \\
\hline & & $\begin{array}{l}\text { Igualmamta } \\
\text { impartante } \\
\end{array}$ & \multicolumn{2}{|r|}{$\begin{array}{c}\text { Importáncia } \\
\text { Frasa }\end{array}$} & \multicolumn{2}{|c|}{\begin{tabular}{c|c} 
Importíncia \\
mogeraga
\end{tabular}} & & \multirow[t]{2}{*}{\begin{tabular}{|c|} 
Importincial \\
Furis
\end{tabular}} & & $\begin{array}{c}\text { Impartincia } \\
\text { mysonsara } \\
\end{array}$ \\
\hline \multicolumn{10}{|c|}{ CRITERIOS PARA O FUNCIONAMENTO DO \$ISTEMA NA FUNDACCAOO } & \\
\hline$\square$ controle de frequencia & L Manuency 30 dos dados pessogis & 1 & 2 & 3 & 4 & 5 & B & 7 & 3) & 9 \\
\hline$\square$ Manutenţ̃o dos dados funcicnais & Contrcle de frequéncia & $\overline{1}$ & $\overline{2}$ & $\sqrt{3}$ & $\overline{4}$ & 5 & $\overline{6}$ & $\overline{7}$ & 8 & 9 \\
\hline$\square$ Controle de frequéncia & $\square$ Manueocría doe afastamentos & 1 & 2 & 3 & 4 & (5) & E & 7 & 8) & 9] \\
\hline 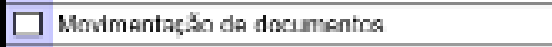 & $\square$ Controla da fremiancla & 1 & {$[2$} & 3] & 4 & [6] & {$[\mathrm{R}$} & ] & 8 & (9) \\
\hline$\square$ Manuiençajo dos dados pesscais & 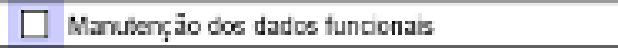 & 1 & 5 & 3 & 4 & 5 & 6 & 7 & 8 & 9 \\
\hline$\square$ Monutiomisos dos afostamsntos & 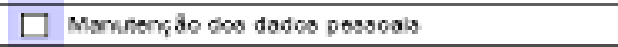 & 1 & {$[2]$} & 3 & 4 & 5 & {$[$ [] } & 7 & a) & (झ) \\
\hline$\square$ Manuten: 30 doe datos pescoals & 口 Movimentor & 1 & 2 & 3 & 4 & 5 & 5 & 7 & 8 & 9] \\
\hline$\square$ Manuren:30 dxa dosoco funcichals & 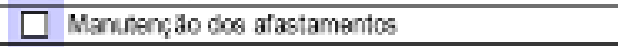 & 1 & 2 & 3 & 4 & 5 & E & 7 & 8 & 9 \\
\hline$\square$ Movimentas 30 de documientos & 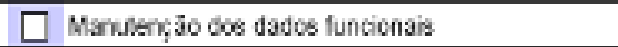 & 1 & 2 & 3 & 4 & 5 & E & 7 & 8 & 9 \\
\hline$\square$ Manulenç3o dos araslamentos & 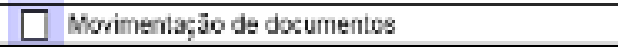 & 1 & 2 & 3 & 4 & 6 & 6 & 7 & 8 & 9 \\
\hline \multicolumn{11}{|c|}{ RFQUUISITOS PARA O CFITÉRIO MANUTFNCĀO DOS DADOS PFSSOAIS } \\
\hline 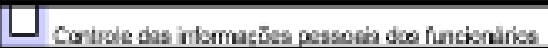 & 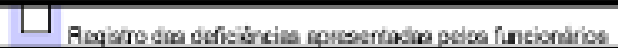 & 1 & 2 & 3 & 4 & 5 & 6 & ? & 무 & प \\
\hline \multicolumn{11}{|c|}{ REQUIIITOE PARA O CRITÉRIO CONTROLE DE FREQUÉACIA } \\
\hline$\square_{\text {Registo de fresulncia }}$ & 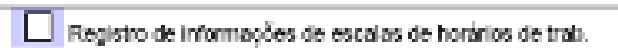 & (1) & 2 & {$[3$} & 4 & 5] & [6 & 7 & 부 & प9 \\
\hline 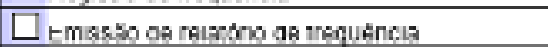 & 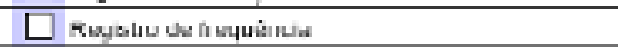 & (1) & 可 & ए & 4 & 5 & [8 & 7 & 0 & D \\
\hline 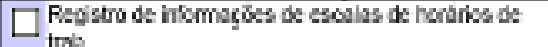 & $\square$ Emikgalo de relatorio de fremuáncla & [1] & [2] & [3] & [4] & [5] & [6] & 7 & ㅂ] & [9] \\
\hline \multicolumn{11}{|c|}{ REOUISTOSOS PARA O CRITERIO MANUTENCCAO DOS DADOS FUNCIONAIS } \\
\hline 口Emissiode & 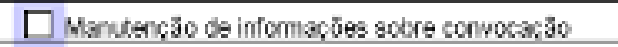 & 1 & 2 & 3 & 4 & 5 & [E & 7 & 8 & 9 \\
\hline 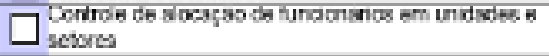 & $\square$ Emissiád de crachàs & 1 & 2 & 3 & 4 & 5 & 6 & 7 & 圆 & 可 \\
\hline 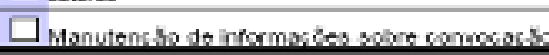 & 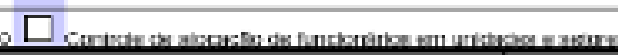 & 1 & 2] & 3] & 4 & 5 & 6 & 7 & . & a \\
\hline \multicolumn{11}{|c|}{ REQUIISITOS PARA O CRITERIO MANUTENCGAO DOS AFASTAMENTOS } \\
\hline 口 Lontroe de rence & 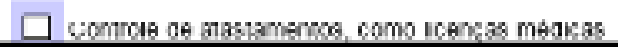 & i & 2 & 3 & [a] & (B) & 0 & $\pi$ & a & 3 \\
\hline \multicolumn{11}{|c|}{ REQUISTTOS PARA O CRITÉRIO MOVIMENTAÇÄO DE DOCUMENTOS } \\
\hline 口 Enrada e saida de CTPS & 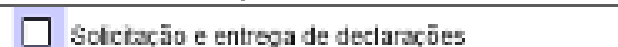 & 1 & 2 & 3 & 4 & 5 & 6 & 7 & 8 & 9 \\
\hline
\end{tabular}

